\title{
A Sub-Optimal Channel Switching-Aware Spectrum Aggregation Approach for CRNs
}

\author{
(Invited Paper) \\ Haeyoung Lee, Seiamak Vahid, and Klaus Moessner \\ Centre for Communication Systems Research (CCSR), \\ Department of Electronic Engineering, University of Surrey, U.K. \\ Email: \{Haeyoung.Lee, S.Vahid, K.Moessner\}@ surrey.ac.uk
}

\begin{abstract}
We consider a cognitive radio network (CRN) that intends to opportunistically aggregate and utilize spectrum of a primary network to achieve higher data rates. In such an opportunistic spectrum access, primary user can reclaim a channel used by a secondary transmission. When the secondary transmission is interrupted by a primary transmission, the secondary network needs to switch the channel of the interrupted transmission, resulting in additional delay. When a secondary user accesses more spectrum to increase its data rate, channel switching could be more frequent. In this context, we formulate a dynamic spectrum aggregation optimisation problem to minimize channel switching delay. While considering multiple users, the problem is formulated as a sum of fractional programming problems. We propose a sub-optimal algorithm that simplifies the fractional programming to linear programming first and solves each linear programming (for each user) using Dinkelbach's algorithm. Simulation results demonstrate that the proposed algorithm can reduce the channel switching delay. When multiple users are served in the network, the proposed algorithm also shows good performance in terms of fairness and total data transmission time.

Index Terms-Cognitive radio networks, spectrum aggregation, channel switching, fractional programming
\end{abstract}

\section{INTRODUCTION}

Opportunistic spectrum access in cognitive radio networks, which enables secondary users (SUs) to utilize the unoccupied spectrum of the primary users (PUs), is regarded as a promising solution to resolve spectrum scarcity and underutilization [1]. In opportunistic spectrum access, given that PUs have higher priorities than SUs for accessing the channels, PUs can access the channels of SUs at any time. When a PU requests to transmit over a channel used by an SU, the SU needs to pause ongoing transmission and to hand over the reclaimed channel to the PU. The SU will then resume its data transmission over a different free channel [2]. Such a channel switching naturally causes additional delay to SU transmission. Moreover, PU can suffer from short-term interference before being detected by the SU [3].

When an SU requires higher data rates, the SU can use multiple channels simultaneously through spectrum aggregation [4]. The more spectrum the SU uses, the higher is the throughput it can achieve. However, the use of extra spectrum implies more frequent channel switching; indeed, in wider spectrum, PUs are likely to reclaim parts of the spectrum more quickly, and, hence, SUs would have to switch more often to a different set of spectrum [5]. Thus, when an SU uses spectrum aggregation, i.e., multiple channels, channel switching issues are likely to be severe and, hence should be properly addressed.

In literature, many research contributions on spectrum aggregation in opportunistic spectrum access have so far focused mainly on how to improve the spectrum efficiency for the cognitive radio network. In [6], the channel capacity obtained through spectrum aggregation are investigated based on a mathematical modelling. In addition, in [7] [8], the research focuses on theoretical analysis of spectrum aggregation for the dynamic traffic, in order to accommodate more secondary users and to improve the data rates. In [9] [10], while considering the hardware constraint for spectrum aggregation, aggregation algorithms with only the aim to achieve higher network throughputs have been proposed. Although the channel switching issue is investigated for spectrum aggregation in [11], the works are about optimal bandwidth selection for aggregation with the assumption of homogeneous channel quality and primary traffic pattern for different channels. To the best our knowledge, spectrum aggregation algorithms for opportunistic spectrum access that decide which channels should be allocated to SUs to reduce channel switching have yet to be proposed.

To this end, we propose in this paper a sub-optimal spectrum aggregation algorithm to allocate multiple idle channels to SUs to reduce the channel switching delay. In order to avoid frequent channel switching, the spectrum aggregation problem is formulated to find the preferable set of channels which have longer idle periods and better channel quality at the same time. Since the formulated problem is expressed in terms of sum of fractional programmings for multiple SUs, we propose a suboptimal algorithm that simplifies the fractional programming into a linear programming and solves each linear programming problem (for single user) with Dinkelbach's algorithm [12].

The rest of this paper is organized as follows. Section II presents the spectrum-usage patterns of the primary network, spectrum access by secondary network, and channel model. The spectrum aggregation optimisation problem to minimize channel switching delay is formulated in Section III. The proposed solution is given in Section IV. After showing simulation results in Section $\mathrm{V}$, our conclusions are summarized in Section VI. 


\section{Models And Assumptions}

\section{A. Spectrum Occupancy Pattern and Spectrum Opportunity}

We consider a cognitive radio network which coexists with a primary network. In the primary network, a base station $\left(B S_{p}\right)$ communicates with PUs through $M$ licensed channels having bandwidth, $B_{n}, n \in\{1,2, \cdots, M\}$.

At any moment, each channel can either be in a busy or idle state, i.e., a PU occupies or not the channel, respectively. The PU's activity in each channel is modelled as an ONOFF process. For channel $n$, ON(busy) and OFF(idle) period, $T_{n}^{B}$ and $T_{n}^{I}$, are assumed to follow an exponential distribution with mean $1 / \lambda_{n}^{B}$ and $1 / \lambda_{n}^{I}$, respectively [13]. The PU's traffic parameters of different channels are assumed to be different, i.e., $\lambda_{v}^{B} \neq \lambda_{w}^{B}$ and $\lambda_{v}^{I} \neq \lambda_{w}^{I}$ for $v \neq w$ and $\forall v, w$ [14].

The PUs, as the licensed users, have priority over the SUs in accessing the channels and the cognitive radio network (as the secondary network) opportunistically detects and exploits idle channels. That is, the SUs can use channel $n$ during the OFF period, $T_{n}^{I}$, governed by its probability density function (PDF), $f_{T_{n}^{I}}(x)$, which can be given as

$$
f_{T_{n}^{I}}(x)=\lambda_{n}^{I} e^{-\lambda_{n}^{I} x} \text { for } x \geq 0, \forall n .
$$

The cumulative distribution function (CDF) of $T_{n}^{I}$ is

$$
F_{T_{n}^{I}}(x)=1-e^{-\lambda_{n}^{I} x} \text { for } x \geq 0, \forall n .
$$

For higher data rates, SUs can use multiple idle channels simultaneously through spectrum aggregation. In order to evaluate spectrum opportunity for the case of spectrum aggregation, we utilise the concept of $N$-channel holding time, $T^{H}(N)$, where $N$ is the number of selected channels for spectrum aggregation [11]. It is defined as the time an SU can simultaneously transmit on selected $N$ channels, i.e., the time between the start of an SU transmission over $N$ channels and PUs accessing one of theses $N$ channels. Therefore, when $N$ channels (e.g., from channel 1 to $N$ ) are selected, $T^{H}(N)$ is the minimum residual idle time of $N$ channels which can be given by $T^{H}(N)=\min \left(T_{1}^{r}, T_{2}^{r}, \cdots, T_{N}^{r}\right)$, where $T_{n}^{r}$ is the residual idle time of channel $n$. Fig. 1 shows an example. When $\mathrm{CH}_{1}, \mathrm{CH}_{2}$ and $\mathrm{CH}_{3}$ are selected by an SU for performing spectrum aggregation at $t_{s}$, channels can only be exploited by the SU during $T_{1}^{r}$, the minimum residual idle time of all three channels in Fig. 1.

According to the renewal theory, PDF of $T_{n}^{r}$ can be derived with the distribution of $T_{n}^{I}$ as follows [15]

$$
f_{T_{n}^{r}}(x)=\left(1-F_{T_{n}^{I}}(x)\right) / E\left[T_{n}^{I}\right]
$$

where $E[\cdot]$ stands for the expectation operator. By using (1) and $E\left[T_{n}^{I}\right]=1 / \lambda_{n}^{I}$ in (2), it is found that $T_{n}^{r}$ also follows an exponential distribution. Since $T^{H}(N)$ is the minimum of $T_{n}^{r}$ where $n \in\{1,2, \cdots, N\}$ that follows an exponential distribution, we obtain that $T^{H}(N)$ is also exponentially distributed with $E\left[T^{H}(N)\right]=1 / \lambda^{H}[16] . E\left[T^{H}(N)\right]$ can be obtained by

$$
E\left[T^{H}(N)\right]=1 / \lambda^{H}=1 /\left(\lambda_{1}^{I}+\cdots+\lambda_{N}^{I}\right) .
$$

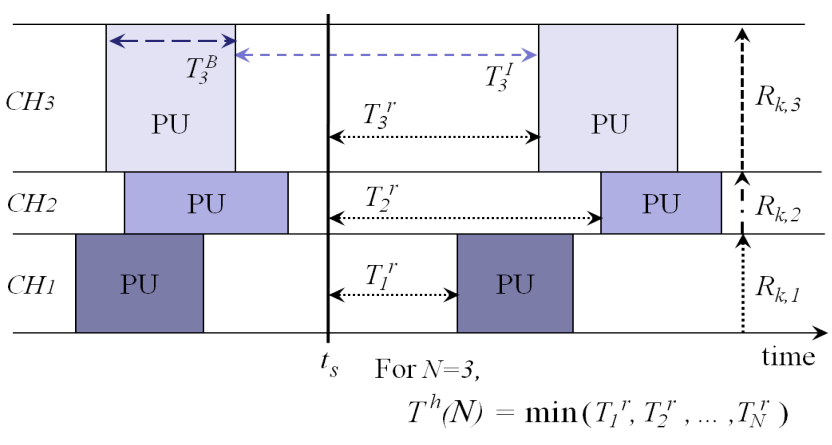

Fig. 1. An example of the use of 3 channels by spectrum aggregation

In the special case that each channel's idle time is independent and identically distributed (i.i.d.), we have $E\left[T^{H}(N)\right]=$ $E\left[T_{n}^{I}\right] / N$. Generally speaking, $E\left[T^{H}(N)\right]$ is decreasing with $N$. The larger the number of channels an SU accesses, the lower the channel holding time is.

\section{B. Cognitive Radio Network and Channel Model}

Suppose that in a cognitive radio network, the base station $\left(B S_{s}\right)$ is able to sense and identify the availability of channels during a sensing duration, $T_{s}$, in Fig. 2. The statistical information on PUs' channel usage pattern is assumed to be stored in a database such as Radio Environment Map (REM) [17] and $B S_{s}$ can access the database. With the information on channel usage pattern from the database and identified idle channels, $B S_{s}$ aggregate and allocate $N$ idle channels to communicate with $K$ SUs. Since multiple channels compose an aggregate channel, hereafter, the term 'channel' can be interchangeable with 'sub-channel' (of an aggregate channel).

While $B S_{s}$ communicates with an SU using multiple subchannels, $B S_{s}$ prefers to utilise allocated sub-channels as long as possible. That is, after $B S_{s}$ checks the sub-channel status every sensing period, $T_{p}$, in Fig. 2 , it keeps using the same sub-channels for the transmission unless the appearance of PU is detected in the allocated sub-channels. However, if any of sub-channels is reclaimed by $\mathrm{PU}$, the transmission is stopped and $B S_{s}$ will allocate new idle su-channels to resume the transmission, i.e., channel switching takes place, resulting in additional delay. We assume that such channel switching delay, $T_{s w}$, in Fig. 2, which encompasses both channel evacuation and link setup times, is a constant value [11] [18].

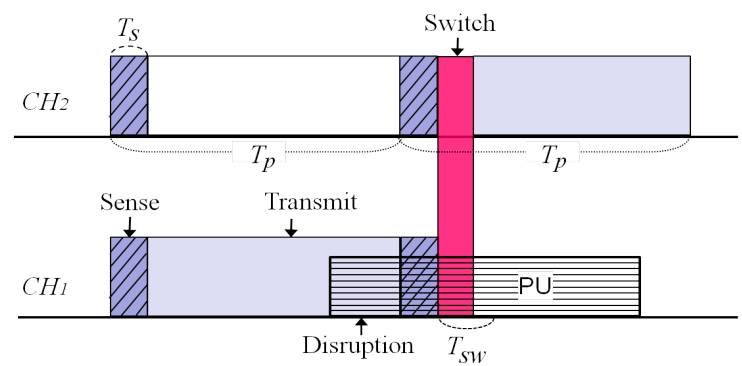

Fig. 2. Spectrum sensing, data transmission and channel switching in a CRN 
Suppose that sub-channels between $B S_{s}$ and SUs are independent and suffer Rayleigh flat fading. The channel coefficient for SU $k$ in sub-channel $n$ (denoted by $h_{k, n}$ ) is complex Gaussian random variable with zero mean and variance $\sigma_{h_{k, n}}^{2}$, i.e., $h_{k, n} \sim \mathcal{C N}\left(0, \sigma_{h_{k, n}}^{2}\right)$ for $\forall k, n$. Assuming that channel state information (CSI) is perfectly known at the receiver, the received signal to noise ratio (SNR) for $\mathrm{SU} k$ in sub-channel $n$ can be then represented as

$$
\rho_{k, n}=\frac{\left|h_{k, n}\right|^{2} P_{k, n}}{\sigma^{2}}=g_{k, n} P_{k, n}
$$

where $\sigma^{2}$ is the variance of the complex-valued zero-mean additive white Gaussian noise (AWGN) and $g_{k, n}=\left|h_{k, n}\right|^{2} / \sigma^{2}$ is the channel SNR. Notice that in (4), $P_{k, n}$ is the transmit power for sub-channel $n$ of SU $k$, i.e., $p_{k, n}=P_{\text {total }} \times B_{n} / \sum_{i=1}^{N} B_{i}$ in equal power allocation for a given total power, $P_{\text {total }}$.

In order to model the available data rate, we use Shannon capacity. By using (4) and knowing that $C_{k, n}=B_{n} \log _{2}(1+$ $\rho_{k, n}$ ) [19], the data rate of sub-channel $n$ for SU $k$ can be given by

$$
C_{k, n}\left(g_{k, n}\right)=B_{n} \cdot \log _{2}\left(1+g_{k, n} P_{k, n}\right) .
$$

From (5), the average data rate can be obtained by taking the expectation of (5) with respect to $g_{k, n}$ for a given $P_{k, n}$. When $N$ sub-channels (e.g., from sub-channel 1 to $N$ ) are selected for aggregation for SU $k$, the average data rate of the aggregate channel, $R_{k}$ can be expressed as the sum of the average data rate of the selected sub-channel $n, R_{k, n}$, where $n \in\{1,2, \cdots, N\}$ as follows

$$
R_{k}=\sum_{i=1}^{N} R_{k, n}=\sum_{i=1}^{N} B_{n} E\left[\log _{2}\left(1+g_{k, n} P_{k, n}\right)\right] .
$$

For instance, in the example of Fig. $1, R_{k}=R_{k, 1}+R_{k, 2}+$ $R_{k, 3}$.

\section{PROBLEM FORMULATION}

We study the problem of spectrum aggregation by properly selecting a subset of sub-channels among the total idle subchannels for the secondary transmission and aim to minimise the channel switching delay for transmission of a given amount of data. Since the switching delay for each switching is assumed to be constant, the minimisation of the total switching delay can be performed by minimising channel switching times. For simplicity, let us consider the case of a single SU to formulate the problem to minimise channel switching times.

Assuming that $B S_{s}$ has an amount of data to transmit towards SU $k, D_{k}^{t x}$, the number of channel switching expected during transmission of $D_{k}^{t x}$ (denoted by $\eta_{k}^{s w}$ ), can be expressed as [11]

$$
\eta_{k}^{s w}=\left[\begin{array}{cl}
\left\lfloor T_{k}^{t x} / M\left[T_{k}^{H}\right]\right\rfloor+1, & \text { if } T_{k}^{t x}>M\left[T_{k}^{H}\right] \\
0, & \text { otherwise }
\end{array}\right],
$$

where $T_{k}^{t x}$ is the required time to transmit the data of the size $D_{k}^{t x}$ and $M\left[T_{k}^{H}\right]$ is the mean holding time of aggregate channels selected sequentially (due to channel switching). When an aggregate channel is selected $\eta_{k}^{s w}+1$ times for $\eta_{k}^{s w}$ times of channel switching, $M\left[T_{k}^{H}\right]$ is the mean of holding time of $\eta_{k}^{s w}+1$ aggregate channels. (i.e., $M\left[T_{k}^{H}\right]$ can be expressed as the average of $E\left[T_{k}^{H}\right]$, the holding time of an aggregate channel for SU $k$ in (3)). Notice that $\lfloor x\rfloor$ denotes the largest integer not larger than $x$. Although there are two cases in (7), the second case that $M\left[T_{k}^{H}\right]$ is larger than $T_{k}^{t x}$, i.e., when channel switching might not be needed, is beyond our interests. Hereinafter, we consider only the first case that $T_{k}^{t x}$ is larger than $E\left[T_{k}^{H}\right]$ in (7). $T_{k}^{t x}$ can be denoted as follows

$$
T_{k}^{t x}=D_{k}^{t x} / E\left[R_{k}\right]
$$

where $E\left[R_{k}\right]$ is the average data rate from $\eta_{k}^{s w}+1$ selected aggregate channels for the whole data transmission (for $D_{k}^{t x}$ ). By inserting (8) into (7), $\eta_{k}^{s w}$ can be re-expressed as

$$
\eta_{k}^{s w}=\left\lfloor D_{t x} /\left(E\left[R_{k}\right] \cdot M\left[T_{k}^{H}\right]\right)\right\rfloor+1 .
$$

From (9), it can be easily see that in order to minimize $\eta_{k}^{s w}$, it is necessary to increase $E\left[R_{k}\right] \cdot M\left[T_{k}^{H}\right]$, which corresponds to the average achievable amount of data to transmit for holding time of an aggregate channel. Increase of $E\left[R_{k}\right] \cdot M\left[T_{k}^{H}\right]$ can be implemented by maximizing $R_{k} \cdot E\left[T_{k}^{H}\right]$ whenever subchannels are newly allocated for channel switching. Then, the problem to minimize $\eta_{k}^{s w}$ can be transformed to maximize $R_{k} \cdot E\left[T_{k}^{H}\right]$.

When $B S_{s}$ has $K$ SUs for channel switching for $N$ idle sub-channels, by using (6) and (3), the spectrum aggregation problem to minimise channel switching can be formulated as follows

$$
\begin{gathered}
\max _{a_{k, n}} \sum_{k=1}^{K} \frac{\sum_{n=1}^{N} a_{k, n} R_{k, n}}{\sum_{n=1}^{N} a_{k, n} \lambda_{n}^{I}} . \\
\text { s.t. } \quad a_{k, n}=\{0,1\}, \forall k, n . \\
\quad \sum_{k=1}^{K} a_{k, n} \leq 1, \forall n .
\end{gathered}
$$

Let $a_{k, n}$ be a binary variable denoting whether or not subchannel $n$ is assigned to the transmission with SU $k$, such that $a_{k, n}$ equals one or zero if sub-channel $n$ is assigned or not, respectively, for SU $k$. In addition, a sub-channel can be used by only one SU at the same time as shown in (11).

Since equal power allocation is utilised here, the use of more sub-channels will lead to achieve higher data rates. However, the holding time of an aggregate channel is likely to become shorter when incorporating more sub-channels in the aggregation process. In addition, since sub-channels with higher SNR can have shorter idle time and/or sub-channels of lower SNR can have longer idle time, two characteristics of sub-channels (i.e., channel quality and idle time distribution) should be considered at the same time in order to find the best set of sub-channels for (10). The preferred set of sub-channels is likely to be composed of sub-channels with high SNR and long idle time while the number of sub-channels is small.

For $K$ SUs and $N$ idle sub-channels, there are $K^{N}$ possible sub-channel allocation combinations. For large value of $K$ and $N$, it is prohibitive in terms of computational 
complexity to find the optimal solution by using exhaustive search. However, the objective function (10) includes the ratio of two linear functions. The problem of maximizing the ratio of two linear functions is called fractional programming problem [20] and the fractional programming problem can be solved by adopting Dinkelbach's method [12]. While the problem (10) is expressed as a maximization of the sum of the fractional programming problems, notice that each fractional programming problem is to find sub-channel allocation for each SU. In order to reduce the complexity, thus, by solving the fractional programming problems sequentially (i.e., to find a best set of sub-channels for each SU one by one) by means of Dinkelbach's method method [12], we propose the sub-optimal algorithm for solving (10) in the following section.

\section{The Proposed Algorithm}

While the initial size of data to transmit can be the same for all SUs, at a certain time when channel switching needs to occur for $K$ SUs, the amount of remained data to transmit on SU $k, D_{k}^{r}$, could be different. In the proposed algorithm, we first sort $K$ SUs in descending order according to $D_{k}^{r}$ where $k \in\{1, \cdots, K\}$. As a greedy method, while the proposed algorithm finds the optimal solution for each SU sequentially, fairness between SUs can be the issue. By ordering SUs according to $D_{k}^{r}$, the SUs having larger remained data can have a chance to use better sub-channels (i.e., with good channel quality and long idle time) than SUs having smaller remained data, thus fairness is expected to improve. The operation of the proposed algorithm is described in Algorithm 1.

After sorting SUs, the proposed algorithm finds the optimal subset of sub-channels among a total of $N$ idle sub-channels for aggregation for each SU. For SU $k$, the spectrum aggregation problem can be simplified as follows

$$
\begin{gathered}
\max _{a_{k, n}} \frac{\sum_{n=1}^{N} a_{k, n} R_{k, n}}{\sum_{n=1}^{N} a_{k, n} \lambda_{n}^{I}} . \\
\text { s.t. } a_{k, n}=\{0,1\}, \forall n .
\end{gathered}
$$

Since the fractional programming problem can be solved efficiently as a sequence of linear programming function, let us convert the fractional form in (12) to a linear function form. For simplicity, let $\Lambda=\left(a_{k, 1}, a_{k, 2}, \cdots, a_{k, N}\right)$, $N(\Lambda)=\sum_{n=1}^{N} a_{k, n} R_{k, n}$ and $D(\Lambda)=\sum_{n=1}^{N} a_{k, n} \lambda_{n}^{I}$. Then the optimisation problem in (12) can be rewritten as

$$
\begin{gathered}
(P): \max _{\Lambda} Q(\Lambda)=\frac{N(\Lambda)}{D(\Lambda)} . \\
\text { s.t. } D(\Lambda) \geq 0, \Lambda \in \Omega \subseteq\{0,1\}^{N} .
\end{gathered}
$$

where $\Omega$ is a nonempty feasible set. Using a parametric approach in [20], the fractional optimisation problem $(P)$ in (13) can be solved indirectly by finding the solution to the following associated non-fractional optimisation problem with the same constraints. i.e.,

$$
\begin{gathered}
(\bar{P}): \max _{\Lambda} \bar{Q}(\Lambda, \lambda)=[N(\Lambda)-\lambda D(\Lambda)], \\
\text { s.t. } D(\Lambda) \geq 0, \Lambda \in \Omega \subseteq\{0,1\}^{N},
\end{gathered}
$$

where $\lambda \in R$ is a constant.

Theorem 1. For $D(\Lambda)>0$ where $\Lambda \in \Omega$ and $\Omega$ is a non-empty feasible set, finding the optimal solution, $\Lambda^{*}$, for the problem $(P)$ is equivalent to obtaining $\lambda^{*}$ that yields $\bar{Q}\left(\Lambda, \lambda^{*}\right)=0$; thus, $\lambda^{*}=\frac{N\left(\Lambda^{*}\right)}{D\left(\Lambda^{*}\right)}$ is the optimum solution.

Proof: See Appendix A.

After converting the fractional programming problem (P) in (13) into the linear programming problem $\bar{P}$ in (14), the proposed algorithm now utilise Dinkelbach's algorithm. The Dinkelbach's algorithm generates a sequence of parameter, $\lambda$, converging to $\lambda^{*}$, as it is described in [12]. The algorithm terminates once the objective value of the problem $(\bar{P})$ becomes zero. By solving a sequence of the linear objective function $(\bar{P})$ with the same conditions, we can obtain the optimal solution of the given problem $(P)$. In the single SU case, the proposed algorithm can find the optimal solution. For multiple SUs, the proposed algorithm uses the same approach from Step 5 to 18 in Algorithm 1 for each SU sequentially and finds the subset of idle sub-channels for aggregation.

The complexity of the proposed algorithm depends on the optimisation problem solver in Step 7 and how fast it to converge to $\lambda^{*}$. While any binary linear programming solver can be used, the proposed algorithm solves the problem in (10) with the complexity $\mathcal{O}(K \log (N C)$ ) where $C=$ $\max \left\{\max _{k, n}\left\lfloor R_{k, n}\right\rfloor, \max _{n}\left\lfloor\lambda_{n}^{I}\right\rfloor, 1\right\}$ in the worst case [20] for a given binary linear programming solver.

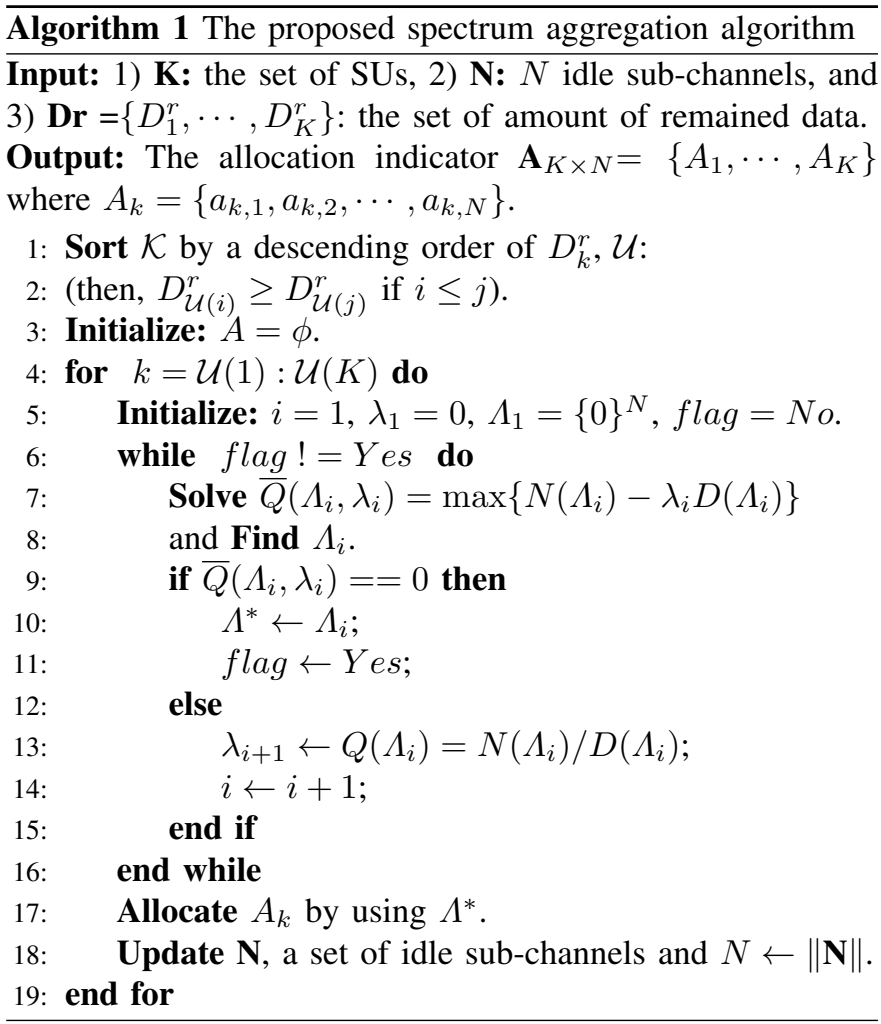


TABLE I

SIMULATION PARAMETERS

\begin{tabular}{|l|l|}
\hline Parameter & Value \\
\hline \hline Sensing duration, $T_{s}$ & $20 \mathrm{~ms}$ \\
\hline Sensing period, $T_{p}$ & $200 \mathrm{~ms}$ \\
\hline Switching delay, $T_{s w}$ & $10 / 20 / 40 \mathrm{~ms}$ \\
\hline Number of total sub-channels & 20 \\
\hline Number of total SUs, $K$ & 3 \\
\hline Amount of data to transmit, $D_{t x}$ & $2 \mathrm{Mbits}$ \\
\hline Total transmit power, $P_{t o t a l}$ & $100 \mathrm{~mW}$ \\
\hline Bandwidth of a sub-channel, $B_{n}$ & $100 \mathrm{kHz}$ \\
\hline Primary user traffic models & $\left(1 / \lambda_{n}^{I}\right.$ and $\left.1 / \lambda_{n}^{B}\right)$ uniformly dist. \\
& in $\left[\mu_{\min }, \mu_{\max }\right]$ \\
\hline Simulation time & $10,000 \mathrm{~s}$ \\
\hline
\end{tabular}

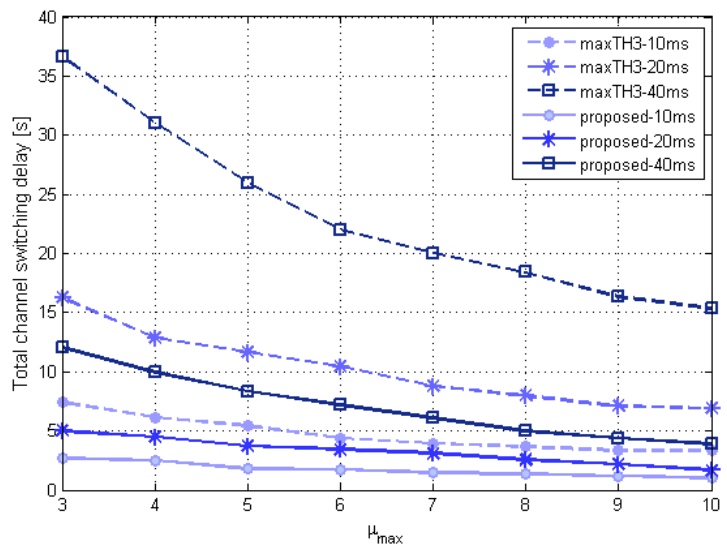

Fig. 3. Total channel switching delay as a function of $\mu_{\max }$.

\section{Simulation Results}

In this section, we present simulation results to evaluate the proposed spectrum aggregation algorithm's performance. Table I summarises the simulation parameters.

For a total of 20 sub-channels, the status of sub-channel $n$ changes based on Exponential On-Off model with $1 / \lambda_{n}^{B}$ and $1 / \lambda_{n}^{I}$. We generate $1 / \lambda_{n}^{B}$ and $1 / \lambda_{n}^{I}$ by using uniform distribution from $\left[\mu_{\min }, \mu_{\max }\right]$, fixing $\mu_{\min }$ to 1 , and varying $\mu_{\max }$ from 3 to 10 [18]. During $T_{s}, B S_{s}$ identifies the subchannel status to check for PU appearances in sub-channels.

Based on statistical information of channel occupancy and identified sub-channels' status, $B S_{s}$ aggregate and allocate idle sub-channels (with better SNR and longer idle time) to SUs for starting or resuming a transmission of 2Mbits of data. While channel switching occurs, various $T_{s w}$ delays of 10, 20, and $40 \mathrm{~ms}$ settings are utilised to evaluate the impact of $T_{s w}$ on total switching delay. In our simulation, we also considered the 'maxTH3'scheme, which aggregates three fixed number of sub-channels with higher SNR; we use it as a reference to compare the performance of our proposed algorithm. The total channel switching delay, the total transmission time and fairness are used for assessing the algorithms' performance.

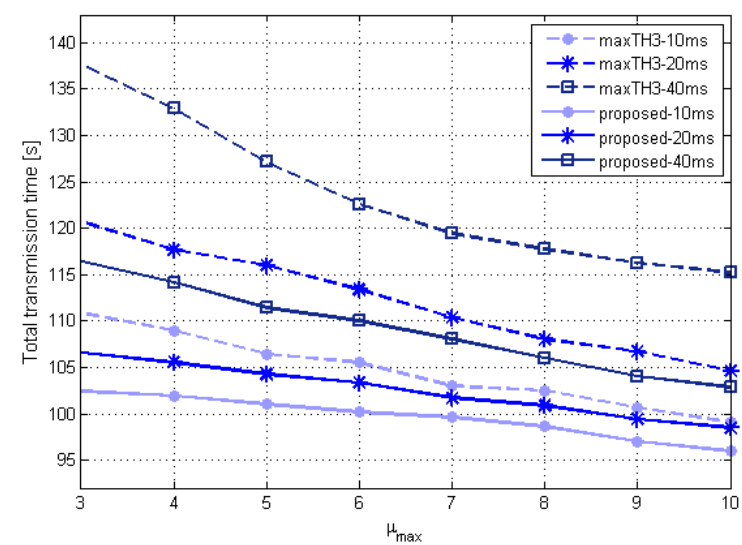

Fig. 4. Data transmission completion as a function of $\mu_{\max }$.

Fig. 3 shows the average total channel switching delay (that SUs will experience for transmission of 2Mbits data) for different $\mu_{\max }$ values. We observe that the total channel switching delay decreases with $\mu_{\max }$ regardless of the algorithm and $T_{s w}$ settings. This is because increasing $\mu_{\max }$ reduces the average rate of primary user activity on sub-channels. The results also indicate that longer $T_{s w}$ generates more channel switching in Fig. 3. At $\mu_{\max }=3$, the transmissions experiences average 7.4 secs switching delay when using 'maxTH3'algorithm and $T_{s w}=10 \mathrm{~ms}$. When $T_{s w}$ is doubled to $20 \mathrm{~ms}$, the average channel switching delay is 16.26 secs, which is more than twice the total switching delay for $T_{s w}=10 \mathrm{~ms}$. We can conclude from this graph that the longer $T_{s w}$ is, the longer the total data transmission time is as well and more channel switching occurs during this prolonged transmission time. In addition, it can be remarked that our proposed algorithm outperforms that the 'maxTH3'algorithm for any $\mu_{\max }$ and $T_{s w}$ values.

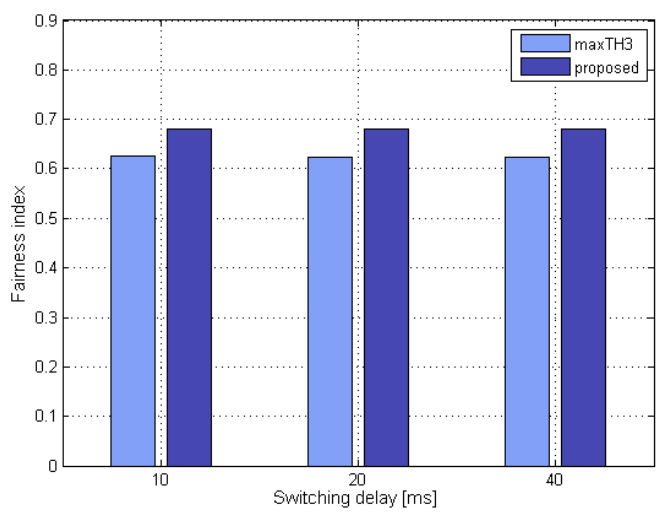

Fig. 5. Jain's Fairness Index of each user's data rates for different channel switching delay, $\mu_{\max }=5$.

Fig. 4 shows the total transmission time for different $\mu_{\max }$ values. It shows that the proposed algorithm can transmit the fixed size of data more quickly than 'maxTH3'for any $T_{s w}$ values. Since the proposed algorithm generates less channel switching delay, it contributes to shorten the data transmission 
completion time. Thus, for a certain time period, the proposed algorithm can help more transmissions to be completed.

Fairness is examined in terms of Jain's fairness index in Fig. 5. Given that the 'maxTH3' is a greedy algorithm, the fixed number of sub-channels with higher SNR are allocated to the SU which arrives earlier. For the SU with later arrival time, it may be that only sub-channels with poor SNR remains and selection of such sub-channel will lead to longer transmission completion time. It can be verified in Fig. 5 that in our proposed algorithm, sorting SUs according to their remaining amount of data yet to be transmitted can be effective for improving fairness.

\section{CONCLUSION}

In this paper, we considered the dynamic spectrum aggregation problem for minimising the channel switching delay in a multiple user context. The spectrum aggregation problem is formulated as a sum of fractional programming problems to maximize achievable data rate for longer holding time by using different channel qualities and different PU channel usage patterns. In order to solve the maximization problem of the sum of fractional programming problems, we solve each fractional programming problems sequentially and propose a sub-optimal algorithm. Simulation results showed that our proposed algorithm produces less channel switching for a fixed amount of transmitted data compared to the reference scheme. By giving a higher priority to SU having a larger size of data to transmit, the proposed algorithm exhibits shorter switching delay and greater fairness, which, in turn, contribute to short data transmission completion time. In this paper, although the PU occupancy of sub-channels is assumed to be independent for different sub-channels, the occupancy of sub-channels could be correlated, especially for adjacent subchannels. The impact of correlation of adjacent sub-channels will be included in our future works.

\section{APPENDIX A}

\section{PROOF OF THEOREM 1} have

Let the vector $\Lambda^{*}$ be the solution for problem $P$, then we

$$
\lambda^{*}=\frac{N\left(\Lambda^{*}\right)}{D\left(\Lambda^{*}\right)} \geq \frac{N(\Lambda)}{D(\Lambda)}, \forall \Lambda \in \Omega .
$$

This leads to

$$
N(\Lambda)-\lambda^{*} D(\Lambda) \leq 0,
$$

which further implies that

$$
\max _{\Lambda}\left\{N(\Lambda)-\lambda^{*} D(\Lambda)\right\}=0
$$

Now if $\Lambda^{*}$ is the optimum solution, then the following holds:

$$
N(\Lambda)-\lambda^{*} D(\Lambda) \leq N\left(\Lambda^{*}\right)-\lambda^{*} D\left(\Lambda^{*}\right)=0
$$

which proves Theorem 1.

\section{ACKNOWLEDGMENT}

This work is performed as part of ACROPOLIS (www.ictacropolis.eu) projects supported by the European Communitys Seventh Framework Program (FP7).

\section{REFERENCES}

[1] Q. Zhao and B. Sadler, "A survey of dynamic spectrum access," IEEE Signal Process. Mag., vol. 24, no. 3, pp. 79-89, May 2007.

[2] I. Christian, S. Moh, I. Chung, and J. Lee, "Spectrum mobility in cognitive radio networks," IEEE Commun. Mag., vol. 50, no. 6, pp. 114-121, June 2012.

[3] B. Wang and K. Liu, "Advances in cognitive radio networks: A survey," IEEE J. Sel. Topics Signal Process., vol. 5, no. 1, pp. 5-23, Feb. 2011

[4] H. Lee, S. Vahid, and K. Moessner, "A survey of radio resource management for spectrum aggregation in lte-advanced," IEEE Commun. Surveys Tuts., to appear in 2014.

[5] S. Dahi and S. Tabbane, "Handoff reducing model for multichannel access in cognitive radio networks," in Proc. IEEE WCNC, Shanghai, China, July 2013, pp. 1780-1785.

[6] L. Jiao, E. Song, V. Pla, and F. Li, "Capacity upper bound of channel assembling in cognitive radio networks with quasistationary primary user activities," IEEE Trans. Veh. Technol., vol. 62, no. 4, pp. 1849-1855, May 2013.

[7] J. Lee and J. So, "Analysis of cognitive radio networks with channel aggregation," in Proc. IEEE WCNC, Sydney, Australia, April 2010.

[8] L. Jiao, F. Li, and V. Pla, "Dynamic channel aggregation strategies in cognitive radio networks with spectrum adaptation," in Proc. IEEE GLOBECOM, Houston, TX, US, Dec. 2011.

[9] J. Lin, L. Shen, N. Bao, B. Su, Z. Deng, and D. Wang, "Channel characteristic aware spectrum aggregation algorithm in cognitive radio networks," in Proc. IEEE LCN, Bonn, Germany, Oct. 2011, pp. 634-639.

[10] D. Chen, Q. Zhang, and W. Jia, "Aggregation aware spectrum assignment in cognitive ad-hoc networks," in Proc. 3rd Int. Conf. CROWNCOM, Singapore, May 2008, pp. 1-6.

[11] D. Xu, E. Jung, and X. Liu, "Optimal bandwidth selection in multichannel cognitive radio networks: How much is too much?" in Proc. 3rd IEEE Int. Symp. DySPAN, Chicago, IL, USA, Oct. 2008, pp. 1-11.

[12] W. Dinkelbach, "On nonlinear fractional programming," Management Science, vol. 13, no. 7, pp. pp. 492-498, 1967. [Online]. Available: http://www.jstor.org/stable/2627691

[13] K. Sriram and W. Whitt, "Characterizing superposition arrival processes in packet multiplexers for voice and data," IEEE J. Sel. Areas Commun., vol. 4, no. 6, pp. 833-846, Sep 1986.

[14] Q. Xiao, Y. Li, M. Zhao, S. Zhou, and J. Wang, "Opportunistic channel selection approach under collision probability constraint in cognitive radio systems," Computer Communications, vol. 32, no. 18, pp. 1914 1922, 2009. [Online]. Available: http://www.sciencedirect.com/science/ article/pii/S0140366409001753

[15] D. Cox, Renewal Theory, ser. Methuen's monographs on applied probability and statistics. Methuen, 1962.

[16] A. W. Marshall and I. Olkin, "A multivariate exponential distribution," Journal of the American Statistical Association, vol. 62, no. 317, pp. 30-44, 1967.

[17] Y. Zhao, L. Morales, J. Gaeddert, K. Bae, J.-S. Um, and J. Reed, "Applying radio environment maps to cognitive wireless regional area networks," in Proc. 2nd IEEE Int. Symp. DySPAN, Dublin, Ireland, April 2007.

[18] L. Yang, L. Cao, and H. Zheng, "Proactive channel access in dynamic spectrum networks," Physical Communication, vol. 1, no. 2, pp. 103 111, 2008. [Online]. Available: http://www.sciencedirect.com/science/ article/pii/S1874490708000268

[19] J. G. Proakis and M. Salehi, Digital Communications 5th Edition. McGraw Hill, 2007.

[20] T. Matsui, Y. Saruwatari, and M. Shigeno, "An analysis of dinkelbach's algorithm for 0-1 fractional programming problems," 1992. 